\title{
Analysis of Switched and Hybrid Systems - Beyond Piecewise Quadratic Methods ${ }^{1}$
}

\author{
Stephen Prajna, Antonis Papachristodoulou \\ Control and Dynamical Systems \\ California Institute of Technology \\ Pasadena, CA 91125 - USA \\ \{prajna, antonis\}@cds.caltech.edu
}

\begin{abstract}
This paper presents a method for stability analysis of switched and hybrid systems using polynomial and piecewise polynomial Lyapunov functions. Computation of such functions can be performed using convex optimization, based on the sum of squares decomposition of multivariate polynomials. The analysis yields several improvements over previous methods and opens up new possibilities, including the possibility of treating nonlinear vector fields and/or switching surfaces and parametric robustness analysis in a unified way.
\end{abstract}

\section{Introduction}

Many systems have dynamics that are described by a set of continuous time differential equations in conjunction with a discrete event process. Such systems are usually referred to as switched or hybrid systems. Stability analysis of switched and hybrid systems has been treated e.g. in $[8,2,5,4]$. See also [3] for a recent survey of the field.

One way of proving stability of switched and hybrid systems is by using piecewise quadratic Lyapunov functions $[8,5,4]$, which are constructed by concatenating several quadratic Lyapunov-like functions. This approach is quite effective, as the search for such Lyapunov functions can be performed by solving linear matrix inequalities (LMIs). However, in some cases it can be conservative.

The present paper provides a new approach to stability analysis of switched and hybrid systems. For proving stability, polynomial and piecewise polynomial Lyapunov functions are constructed using the sum of squares decomposition [7], which can be efficiently computed using semidefinite programming, e.g. using the software [9]. The method generalizes previous analysis methods using quadratic and piecewise quadratic Lyapunov functions. Some features of the new approach are:

${ }^{1}$ This work was supported by AFOSR "Unified Theory for Complex Biological and Engineering Networks", NIH/NIGMS AfCS (Alliance for Cellular Signalling), DARPA "Enlightened Multiscale Simulation of Biochemical Networks", the Kitano ERATO Systems Biology Project, and URI "Protecting Infrastructures from Themselves".
- it provides a less conservative test for proving stability under arbitrary switching,

- stability can be proven with a smaller number of Lyapunov-like functions, eliminating the need of refining the state space partition,

- the method can be applied to systems with nonlinear subsystems and nonlinear switching surfaces,

- parametric robustness analysis can be performed in a straightforward manner.

\section{Preliminaries}

\subsection{Switched and Hybrid Systems}

In this section, we present some preliminaries on switched and hybrid systems, and establish the notation for later use. The systems considered in this paper are of the following form:

$$
\dot{x}=f_{i}(x), \quad i \in I=\{1, \ldots, N\},
$$

where $x \in \mathbb{R}^{n}$ is the continuous state, $i$ is the discrete state, $f_{i}(x)$ is the vector field describing the dynamics of the $i$-th mode/subsystem, and $I$ is the index set. Without loss of generality, we assume that the origin is an equilibrium of the system.

Depending on how the discrete state $i$ evolves, a system like (1) can be categorized as a switched system, if for each $x \in \mathbb{R}^{n}$ only one $i \in I$ is possible, or as a hybrid system, if for some $x \in \mathbb{R}^{n}$ multiple $i$ are possible. The former type of systems includes systems with saturation and variable structure systems, whereas the latter type includes systems with hysteresis, systems with finite automata, etc.

More specifically, in the case of switched systems, the system is in the $i$-th mode at time $t$ if $x(t) \in X_{i}$, where $X_{i} \subset \mathbb{R}^{n}$ is a region of the state space described by

$$
X_{i}=\left\{x \in \mathbb{R}^{n}: g_{i k}(x) \geq 0, \text { for } k=1, \ldots, m_{X_{i}}\right\},
$$

for some $g_{i k}: \mathbb{R}^{n} \rightarrow \mathbb{R}$. Additionally, the state space partition $\left\{X_{i}\right\}$ must satisfy $\bigcup_{i \in I} X_{i}=\mathbb{R}^{n}$ and $\operatorname{int}\left(X_{i}\right) \cap$ $\operatorname{int}\left(X_{j}\right)=\emptyset$ for $i \neq j$. A switching surface between the 
$i$-th and $j$-th modes, i.e. a boundary between $X_{i}$ and $X_{j}$, is given by

$$
S_{i j}=\left\{x: h_{i j 0}(x)=0, h_{i j k}(x) \geq 0, k=1, \ldots, m_{S_{i j}}\right\},
$$

for some $h_{i j k}: \mathbb{R}^{n} \rightarrow \mathbb{R}$. Note that the transition between modes on this surface can occur in both directions. Although in principle the direction of transition for a particular $x \in S_{i j}$ can be determined from the vector fields $f_{i}(x)$ and $f_{j}(x)$, it is assumed in our analysis that such a characterization is not performed a priori.

On the other hand, the evolution of the discrete state in a hybrid system is governed by

$$
i(t)=\phi\left(x(t), i\left(t^{-}\right)\right)
$$

with $\phi: \mathbb{R}^{n} \times I \rightarrow I$. Corresponding to the transition law $\phi$, there exists a region of the state space where a particular mode can be active. For the $i$-th mode, the active region is denoted by $X_{i}$, and is given by ${ }^{1}$

$$
X_{i}=\left\{x \in \mathbb{R}^{n}: g_{i k}(x) \geq 0, \text { for } k=1, \ldots, m_{X_{i}}\right\} .
$$

In the hybrid system case, $\bigcup_{i \in I} X_{i}=\mathbb{R}^{n}$ still holds, but $\operatorname{int}\left(X_{i}\right) \cap \operatorname{int}\left(X_{j}\right)$ is not necessarily empty for $i \neq j$. The transition set from the $j$-th mode to the $i$-th mode in a hybrid system is described by

$$
\begin{aligned}
S_{i j} & =\{x: i=\phi(x, j)\} \\
& =\left\{x: h_{i j 0}(x)=0, h_{i j k}(x) \geq 0, k=1, \ldots, m_{S_{i j}}\right\} .
\end{aligned}
$$

In contrast to switched systems, the transition between modes on $S_{i j}$ for a hybrid system occurs only in one direction, namely from $j$ to $i$.

Throughout the paper, it is assumed that the discrete state $i(t)$ is piecewise continuous. Systems with infinitely fast switching, such as those that have sliding modes, are excluded from our discussion. We also assume that the functions $f_{i}, g_{i k}$, and $h_{i j k}$ are polynomials. For the case in which any of these functions is nonpolynomial, see the comment at the end of Section 3.3.

\subsection{Sum of Squares Decomposition}

Our analysis in the later sections is based on the sum of squares decomposition of multivariate polynomials. A multivariate polynomial $p(x)$ is a sum of squares if there exist polynomials $p_{1}(x), \ldots, p_{m}(x)$ such that $p(x)=$ $\sum_{i=1}^{m} p_{i}^{2}(x)$. This in turn is equivalent to the existence of a positive semidefinite matrix $Q$, and a properly chosen vector of monomials $Z(x)$ such that $p(x)=Z^{T}(x) Q Z(x)$.

What makes the sum of squares decomposition attractive is the fact that such a decomposition can be computed using semidefinite programming, since the computation of $Q$ is nothing but a search for a positive semidefinite matrix subject to some affine constraints. Coupled

\footnotetext{
${ }^{2}$ A notation similar to (2) is chosen here for simplicity; the interpretation should be clear from the context.
}

with the property that $p(x)$ being a sum of squares implies $^{2} p(x) \geq 0$, the sum of squares decomposition provides a computational relaxation for proving polynomial positivity, which belongs to the class of NP-hard problems. Three kinds of polynomial positivity and their corresponding sum of squares computational relaxations are shown in Table 1.

The sum of squares decomposition has been exploited to algorithmically construct Lyapunov functions for nonlinear systems $[7,6]$. For this purpose, real coefficients $c_{1}, \ldots, c_{m}$ are used to parameterize a set of Lyapunov functions in the following way:

$$
\mathcal{V}=\left\{p(x): p(x)=p_{0}(x)+\sum_{i=1}^{m} c_{i} p_{i}(x)\right\}
$$

where $p_{i}(x)$ are some polynomials; for example they could be monomials of degree up to some number. The search for a Lyapunov function $V(x) \in \mathcal{V}$, or equivalently some $c_{i}$, such that $V(x)$ is positive definite and $\frac{d V}{d t}$ is negative definite can still be formulated as a sum of squares problem and solved using semidefinite programming.

For brevity, the theorems in the subsequent sections will be formulated in terms of inequalities such as $V(x) \geq 0$ or $V(x)>0$. In fact, these theorems hold when the inequalities are interpreted in the usual manner. However, if the computation of Lyapunov functions is to be performed using semidefinite programming, then these inequalities have to be interpreted as sum of squares conditions, in the way summarized in Table 1.

\section{Stability Analysis}

\subsection{Stability Under Arbitrary Switching}

We will first consider stability of the system (1) under arbitrary switching. A sufficient condition for such stability is the existence of a global common Lyapunov function for all $f_{i}$ 's, as summarized in the following theorem.

Theorem 1 Suppose that for the set of vector fields $\left\{f_{i}\right\}$ there exists a polynomial $V(x)$ such that $V(0)=0$ and

$$
\begin{aligned}
& V(x)>0 \quad \forall x \neq 0, \\
& \frac{\partial V}{\partial x} f_{i}(x)<0 \quad \forall x \neq 0, i \in I,
\end{aligned}
$$

then the origin of the state space of the system (1) is globally asymptotically stable under arbitrary switching.

Notice in particular that if the vector fields are linear, i.e. $f_{i}(x)=A_{i} x$, and if $V(x)$ is chosen to be quadratic, say $V(x)=x^{T} P x$, then the conditions in Theorem 1 correspond to the well-known LMIs $P>0, A_{i}^{T} P+P A_{i}<0$ for all $i$, which prove quadratic stability of the system. For higher degree polynomial vector fields and Lyapunov

\footnotetext{
${ }^{2}$ Note that the converse implication is true only in special cases. One of such instances is when the polynomial is quadratic.
} 


\begin{tabular}{|l|l|}
\hline Positive semidefinite: $p(x) \geq 0 \quad \forall x \in \mathbb{R}^{n}$. & $p(x)$ is a sum of squares. \\
\hline $\begin{array}{l}\text { Positive definite: } p(x)>0 \quad \forall x \neq 0 ; p(0)=0 . \\
\text { - If } p(x) \text { is homogeneous of degree } N .\end{array}$ & $\left(p(x)-\epsilon \sum_{i=1}^{n} x_{i}^{N}\right)$ is a sum of squares. \\
- If $p(x)$ is of degree $N$, but nonhomogeneous. & $\left\{\begin{array}{l}\left(p(x)-\sum_{i=1}^{n} \sum_{j=1}^{N / 2} \epsilon_{i j} x_{i}^{2 j}\right) \text { is a sum of squares; } \\
\sum_{j=1}^{N / 2} \epsilon_{i j} \geq \epsilon \quad \forall i ; \quad \epsilon_{i j} \geq 0 \quad \forall i, j .\end{array}\right.$ \\
\hline Strict positivity: $p(x)>0 \quad \forall x \in \mathbb{R}^{n}$. & $(p(x)-\epsilon)$ is a sum of squares. \\
\hline
\end{tabular}

Table 1: Three kinds of polynomial positivity (on the left) and the corresponding sum of squares conditions (on the right). Conditions on the right are sufficient for those on the left. The polynomial degree $N$ is assumed to be even, otherwise the polynomial will be negative for some $x$. Here the $\epsilon$ are some positive real numbers.

functions, the search for $V(x)$ can also be performed using semidefinite programming by formulating the conditions as sum of squares conditions, as described in Section 2.2 . The higher degree test is generally less conservative than the quadratic test, as in many cases global higher degree Lyapunov functions exist for systems that do not possess a global quadratic Lyapunov function. At worst, these two tests have the same conservatism.

Example 2 Consider the system $\dot{x}=f_{i}(x), x=$ $\left[\begin{array}{ll}x_{1} & x_{2}\end{array}\right]^{T}$, with

$$
f_{1}(x)=\left[\begin{array}{c}
-5 x_{1}-4 x_{2} \\
-x_{1}-2 x_{2}
\end{array}\right], \quad f_{2}(x)=\left[\begin{array}{c}
-2 x_{1}-4 x_{2} \\
20 x_{1}-2 x_{2}
\end{array}\right] .
$$

It can be proven using a dual semidefinite program that no global quadratic Lyapunov function exists for this system [5]. Nevertheless, a global sextic Lyapunov function

$$
\begin{aligned}
V(x)= & 19.861 x_{1}^{6}+11.709 x_{1}^{5} x_{2}+14.17 x_{1}^{4} x_{2}^{2} \\
& +4.2277 x_{1}^{3} x_{2}^{3}+8.3495 x_{1}^{2} x_{2}^{4}-1.2117 x_{1} x_{2}^{5} \\
& +1.0421 x_{2}^{6}
\end{aligned}
$$

exists, and therefore the system is asymptotically stable under arbitrary switching (cf. Figure 1).

\subsection{Piecewise Polynomial Lyapunov Functions}

Most switched and hybrid systems come with a prescribed switching scheme or a discrete transition rule. In this case, there is no need to use the global analysis method presented in Section 3.1. Stability can be proven in a more effective way using piecewise polynomial Lyapunov functions. Such functions are patched from several polynomial functions $V_{i}(x)$ (also termed Lyapunovlike functions), typically corresponding to the state space partition $\left\{X_{i}\right\}$. The Lyapunov-like function $V_{i}(x)$ and its time derivative along the trajectory of the $i$-th mode are required to be positive and negative respectively, only within $X_{i}$.

The conditions in the previous paragraph can be accommodated using a method similar to the S-procedure [1] as follows. To incorporate the fact that $V_{i}(x)$ only needs to be positive on $X_{i}$, where $X_{i}$ is described by (2), we impose the relaxed condition

$$
V_{i}(x)-\sum_{k=1}^{m x_{i}} a_{i k}(x) g_{i k}(x)>0
$$

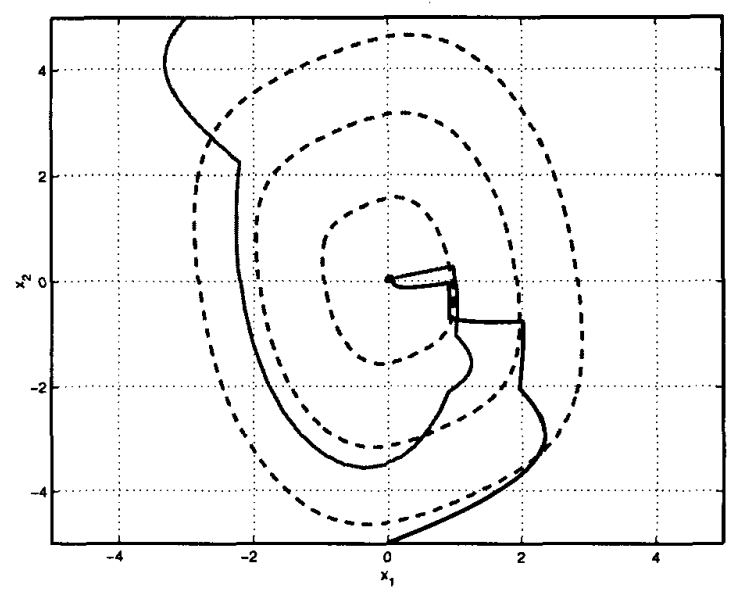

Figure 1: Trajectories of the system in Example 2 under arbitrary switching. Dashed curves are level curves of the common Lyapunov function.

for some $a_{i k}(x) \geq 0$. Since $g_{i k}(x)$ is nonnegative on $X_{i}$, the above condition implies that $V_{i}(x)$ is positive on $X_{i}$. An analogous condition can be imposed on $\frac{d V_{i}}{d t}$. Note that there is no requirement in our method that the multipliers $a_{i k}(x)$ be constants (as in the S-procedure); they can also be polynomials of higher degree. Thus, our condition is generally less conservative than the S-procedure.

3.2.1 Switched Systems: As mentioned in the preliminaries, for a switched system the transition between modes on a switching surface is not characterized a priori. Without characterizing the direction of switching, it is essential that the piecewise Lyapunov function used to prove stability be continuous on every $S_{i j}$. For $S_{i j}$ as in (3), imposing $V_{i}(x)+c_{i j}(x) h_{i j 0}(x)-V_{j}(x)=0$, where $c_{i j}(x)$ is an arbitrary polynomial, will guarantee the continuity of $V(x)$ on $S_{i j}$. To this end, we have the following theorem for switched systems.

Theorem 3 Consider the switched system (1)-(3). Assume that there exist polynomials $V_{i}(x), c_{i j}(x)$, with $V_{i}(0)=0$ if $0 \in X_{i}$, and sums of squares $a_{i k}(x) \geq 0$, $b_{i k}(x) \geq 0$, such that

\footnotetext{
${ }^{3}$ The theorem actually holds as long as $a_{i k}(x)$ and $b_{i k}(x)$ are nonnegative, even if they are not sums of squares (cf. footnote 2 on the previous page and the remark at the end of Subsection 2.2).
} 


$$
\begin{aligned}
& V_{i}(x)-\sum_{k=1}^{m_{X_{i}}} a_{i k}(x) g_{i k}(x)>0 \quad \forall x \neq 0, i \in I, \\
& \frac{\partial V_{i}}{\partial x} f_{i}(x)+\sum_{k=1}^{m_{X_{i}}} b_{i k}(x) g_{i k}(x)<0 \quad \forall x \neq 0, i \in I, \\
& V_{i}(x)+c_{i j}(x) h_{i j 0}(x)-V_{j}(x)=0 \quad \forall i, j .
\end{aligned}
$$

Then the origin of the state space is globally asymptotically stable. A Lyapunov function that proves this is the piecewise polynomial function $V(x)$ defined by

$$
V(x)=V_{i}(x), \quad \text { if } x \in X_{i} .
$$

Even though the switched system is stable, low degree (e.g. quadratic) $V_{i}(x)$ that satisfy the above conditions may not exist, as those conditions are only sufficient for stability. In this case, an improved test can be performed by dividing the state space into a more refined partition than the original partition $\left\{X_{i}\right\}$, and then constructing a piecewise Lyapunov function (of the same degree as before) based on this new partition. For systems with more than two state variables, this refinement is obviously not an easy matter. A simpler way for obtaining an improved test is to use a higher degree Lyapunov function based on the original partition, as illustrated by the following example.

Example 4 Consider the switched system $\dot{x}=f_{i}(x)$ with four state variables and two modes:

$$
\begin{aligned}
& f_{1}(x)= {\left[\begin{array}{c}
-x_{1}-23 x_{2}+12 x_{3}-2 x_{4} \\
-0.5 x_{1}+8.5 x_{2}-6 x_{3}+0.5 x_{4} \\
0.5 x_{1}+26 x_{2}-9.5 x_{3}+5 x_{4} \\
-3 x_{1}-35 x_{2}+12 x_{3}-6 x_{4}
\end{array}\right], } \\
& f_{2}(x)=\left[\begin{array}{c}
-1.4 x_{1}-18.6 x_{2}+8 x_{3}-1.6 x_{4} \\
-0.3 x_{1}+8.3 x_{2}-4 x_{3}+1.3 x_{4} \\
1.7 x_{1}+20.6 x_{2}-5.7 x_{3}+3.6 x_{4} \\
-3.4 x_{1}-28.6 x_{2}+8 x_{3}-4.6 x_{4}
\end{array}\right], \\
& X_{1}=\left\{x \in \mathbb{R}^{4}: g(x) \geq 0\right\}, \\
& X_{2}=\left\{x \in \mathbb{R}^{4}: g(x) \leq 0\right\},
\end{aligned}
$$

where $g(x)=\left(x_{1}+0.5 x_{2}+1.5 x_{3}+0.5 x_{4}\right)\left(x_{1}-0.5 x_{2}+\right.$ $\left.0.5 x_{3}-0.5 x_{4}\right)$. No piecewise quadratic Lyapunov function (using the original state space partition) exists for this system. Refining the partition for this system is not easy, thus we resort to higher order Lyapunov function instead. A homogeneous piecewise quartic Lyapunov function can be found by solving the optimization problem corresponding to the conditions in Theorem 3. This proves that the origin of the state space is globally asymptotically stable.

3.2.2 Hybrid Systems: Mode transitions in a hybrid system are characterized a priori. Because of this, a piecewise Lyapunov function for a hybrid system need not be continuous, and it is enough to have $V_{i}(x) \leq V_{j}(x)$ on $S_{i j}$. This is taken into account in condition (17) of the theorem below.
Theorem 5 Consider the hybrid system (1), (5)-(6). Assume that there exist polynomials $V_{i}(x), c_{i j}(x)$, and $a_{i k}(x) \geq 0, b_{i k}(x) \geq 0, d_{i j k}(x) \geq 0$, such that $V_{i}(0)=0$ if $0 \in X_{i}$, and

$$
\begin{gathered}
V_{i}(x)-\sum_{k=1}^{m_{X_{i}}} a_{i k}(x) g_{i k}(x)>0 \quad \forall x \neq 0, i \in I, \\
\begin{aligned}
\frac{\partial V_{i}}{\partial x} f_{i}(x)+\sum_{k=1}^{m_{X_{i}}} b_{i k}(x) g_{i k}(x)<0 \quad \forall x \neq 0, i \in I, \\
V_{i}(x)+c_{i j}(x) h_{i j 0}(x)+\sum_{k=1}^{m_{S_{i j}}} d_{i j k}(x) h_{i j k}(x) \ldots \\
-V_{j}(x) \leq 0 \quad \forall i, j .
\end{aligned}
\end{gathered}
$$

Then the origin is globally asymptotically stable.

3.3 Nonlinear Vector Fields and Switching Surfaces/Transition Sets

So far, the systems we have considered in the examples have linear subsystems and linear switching surfaces. As mentioned previously, the sum of squares conditions can be applied directly to systems with nonlinear vector fields and nonlinear switching surfaces or transition sets. To illustrate this, consider the following example.

Example 6 Let the hybrid system $\dot{x}=f_{i}(x)$ be composed from two subsystems

$$
\begin{aligned}
& f_{1}(x)=\left[\begin{array}{c}
-2 x_{1}-x_{1}^{3}-5 x_{2}-x_{2}^{3} \\
6 x_{1}+x_{1}^{3}-3 x_{2}-x_{2}^{3}
\end{array}\right], \\
& f_{2}(x)=\left[\begin{array}{c}
x_{2}+x_{1}^{2}-x_{1}^{3} \\
4 x_{1}+2 x_{2}
\end{array}\right],
\end{aligned}
$$

with its transition rule given by

$$
\begin{aligned}
& \phi(0)=1, \\
& \phi(t)=\left\{\begin{array}{l}
1, \text { if } i(t-)=2 \text { and } x_{2}^{2}(t)=x_{1}^{3}(t), \\
2, \text { if } i(t-)=1 \text { and } x_{2}(t)=0, x_{1}(t) \geq 0 .
\end{array}\right.
\end{aligned}
$$

Figure 2 depicts some trajectories of the system. The active regions corresponding to the two modes are $X_{1}=\mathbb{R}^{2}$ and $X_{2}=\left\{x \in \mathbb{R}^{2}:\left(x_{1}^{3}-x_{2}^{2}\right) \geq 0\right\}$, while the transition sets are $S_{12}=\left\{x \in \mathbb{R}^{2}: x_{2}^{2}=x_{1}^{3}\right\}$ and $S_{21}=$ $\left\{x \in \mathbb{R}^{2}: x_{2}=0, x_{1} \geq 0\right\}$. Using Theorem 5, the origin can be proven globally asymptotically stable with a sextic piecewise polynomial Lyapunov function given by

$$
V(x(t))=V_{i}(x(t)), \quad \text { if } \phi(t)=i,
$$

for some $V_{i}(x)$ 's.

In fact, even for a system with a rational or nonpolynomial vector field, a system embedding can sometimes be made such that a Lyapunov function that proves stability can be computed using the sum of squares decomposition. This has been presented in [6] and will not be discussed in this paper. The same technique can also be applied to nonpolynomial switching surfaces or transition sets.

Proceedings of the American Control Conference 


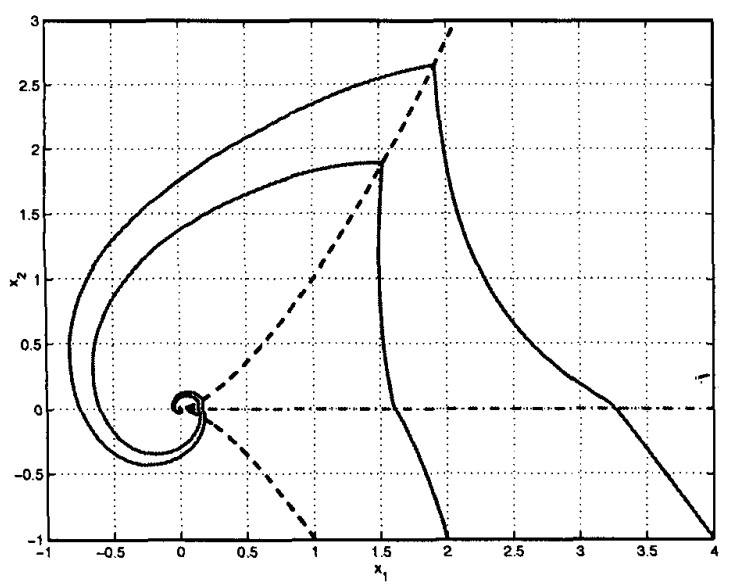

Figure 2: Trajectories of the system in Example 6. Dashdotted line and dashed curves show $S_{21}$ and $S_{12}$, respectively.

\section{Robust Stability Analysis}

In this section we consider robust stability analysis of switched or hybrid systems with uncertainty. Uncertainty in a switched or hybrid system can be present in the vector fields describing the flow of the system and/or in the switching scheme/transition law. The uncertainty can be of parametric nature, or caused by perturbation of the vector field, switching delays, etc.

A method for robustness analysis has been proposed in [8]. The approach is based on bounding $S_{i j}$ by an uncertain switching set, and $X_{i}$ by a bigger set where the corresponding Lyapunov-like function is decreasing. Since this analysis is carried out using conditions similar to those given in Section 3, it can be immediately generalized to make use of polynomial functions. The method is well-suited for robustness analysis with respect to nonparametric uncertainty, but unfortunately, although in principle parametric uncertainty can be handled in a similar fashion, it is not treated in a direct and efficient way.

We now present an analysis technique for handling parametric uncertainty in a direct way, based on parameter dependent Lyapunov-like functions and multipliers. Computation of parameter dependent quadratic Lyapunov-like functions using LMIs had been previously difficult, since such functions are nonquadratic polynomials in the state and parameter variables. Using the sum of squares decomposition, computation of even higher degree functions is straightforward.

Let us denote the uncertain parameters by $p \in \mathbb{R}^{\tilde{n}}$, and let the set of admissible parameters be given by

$$
\begin{gathered}
P=\left\{p \in \mathbb{R}^{\tilde{n}}: q_{k_{1}}(p) \geq 0, k_{1}=1, \ldots, K_{1} ;\right. \\
\left.r_{k_{2}}(p)=0, k_{2}=1, \ldots, K_{2}\right\}
\end{gathered}
$$

for some polynomials $q_{k_{1}}(p)$ and $r_{k_{2}}(p)$. Furthermore, assume that the vector fields $f_{i}$ and polynomials describing $X_{i}$ and $S_{i j}$ depend on $p$. Theorems 1, 3, and 5 can be modified to accommodate parameter dependent Lyapunov functions and multipliers. For brevity, we only present the parameter dependent version of Theorem 5 .

Theorem 7 Consider the hybrid system (1), (5)-(6), which has unknown parameters $p \in P$, where $P$ is as in (18). Assume that there exist polynomials $V_{i}(x, p)$, $c_{i j}(x, p), \hat{a}_{i k}(x, p), \hat{b}_{i k}(x, p), \hat{d}_{i j k}(x, p)$, and $a_{i k}(x, p) \geq 0$, $\tilde{a}_{i k}(x, p) \geq 0, b_{i k}(x, p) \geq 0, \tilde{b}_{i k}(x, p) \geq 0, d_{i j k}(x, p) \geq 0$, $\tilde{d}_{i j k}(x, p) \geq 0$, such that $V_{i}(0, p)=0$ if $0 \in X_{i}$ and Equations (19)-(21) on the next page are satisfied. Then the origin of the state space is robustly asymptotically stable with respect to the unknown parameters $p \in P$.

Example 8 Let us consider the hybrid system $\dot{x}=f_{i}(x)$, with vector fields

$$
f_{1}(x)=\left[\begin{array}{c}
-x_{1}-100 x_{2} \\
10 x_{1}-x_{2}
\end{array}\right], \quad f_{2}(x)=\left[\begin{array}{c}
x_{1}+10 x_{2} \\
-100 x_{1}+x_{2}
\end{array}\right]
$$

and transition law

$$
\begin{aligned}
& i(0)=1, \\
& i(t)= \begin{cases}2, & \text { if } i(t-)=1 \text { and }-p x_{1}(t)-x_{2}(t)=0, \\
1, & \text { if } i(t-)=2 \text { and } 2 x_{1}(t)-x_{2}(t)=0 .\end{cases}
\end{aligned}
$$

Notice the dependence of the first switching surface on the unknown parameter $p \in \mathbb{R}$. Stability of the system depends on the value of $p$. In this example, we have deliberately chosen a system with linear subsystems, so that robust stability of the system can also be analyzed in a purely analytical way for comparison purposes. By computing the flows of the subsystems, it can be proven that the system is stable for $p>2.165$ and unstable for $p<2.163$. At $p \simeq 2.164$ it exhibits a limit cycle (see Figure 3). With parameter dependent Lyapunov-like functions of the form

$$
V_{i}(x, p)=V_{i, 1}(x)+p V_{i, 2}(x)
$$

robust stability of the system with respect to $p \in P=$ $\{p: p-C \geq 0\}$, where $C$ is a constant, can be proven. Using quadratic $V_{i, 1}(x)$ and $V_{i, 2}(x)$, we can prove robust stability for $C=5.86$. Tighter robustness bounds can be obtained by increasing the degree of the Lyapunov-like functions, as depicted in Table 2.

\section{Conclusions}

A new method for stability analysis of switched and hybrid systems has been presented. The method is based on polynomial and piecewise polynomial Lyapunov functions, whose computation can be efficiently performed using the sum of squares decomposition and semidefinite programming. 


$$
\begin{aligned}
& V_{i}(x, p)-\sum_{k=1}^{m_{X_{i}}} a_{i k}(x, p) g_{i k}(x, p)-\sum_{k=1}^{K_{1}} \tilde{a}_{i k}(x, p) q_{k}(p)-\sum_{k=1}^{K_{2}} \hat{a}_{i k}(x, p) r_{k}(p)>0 \quad \forall x \neq 0, i \in I \\
& \frac{\partial V_{i}}{\partial x} f_{i}(x, p)+\sum_{k=1}^{m_{X_{i}}} b_{i k}(x, p) g_{i k}(x, p)+\sum_{k=1}^{K_{1}} \tilde{b}_{i k}(x, p) q_{k}(p)+\sum_{k=1}^{K_{2}} \hat{b}_{i k}(x, p) r_{k}(p)<0 \quad \forall x \neq 0, i \in I \\
& V_{i}(x, p)+c_{i j}(x, p) h_{i j 0}(x, p)+\sum_{k=1}^{m_{S_{i j}}} d_{i j k}(x, p) h_{i j k}(x, p)-V_{j}(x, p)+\sum_{k=1}^{K_{1}} \tilde{d}_{i j k}(x, p) q_{k}(p)+\sum_{k=1}^{K_{2}} \hat{d}_{i j k}(x, p) r_{k}(p) \leq 0 \quad \forall i, j .
\end{aligned}
$$

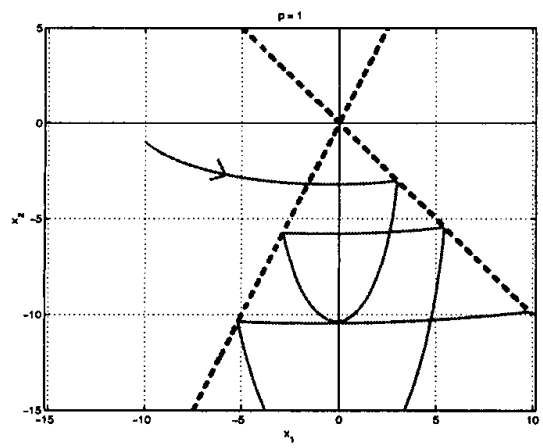

(a) $p=1$

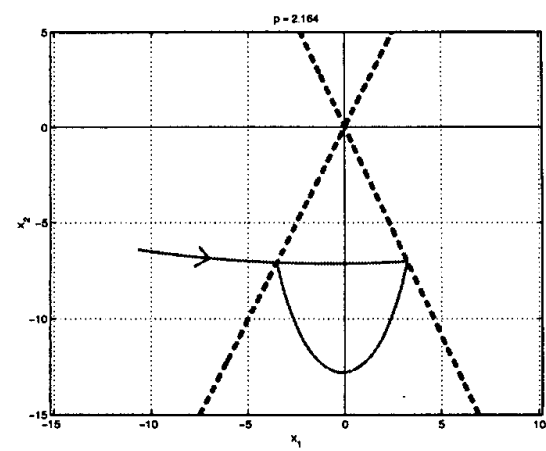

(b) $p \simeq 2.164$

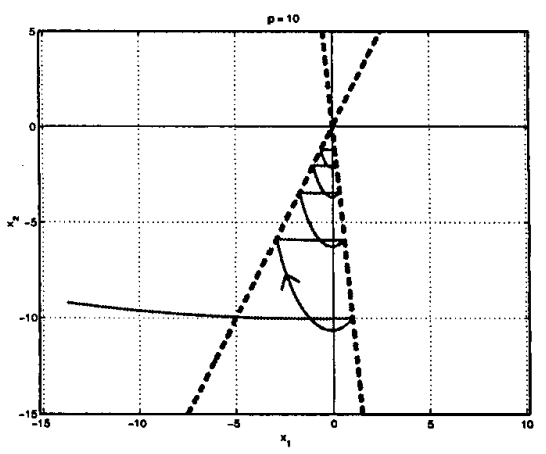

(c) $p=10$

Figure 3: Trajectories of the system in Example 8 for different values of $p$. Dashed lines represent the transition sets.

\begin{tabular}{|c|c|}
\hline Degree of $V_{i, k}(x)$ & $C$ \\
\hline 2 & 5.86 \\
4 & 2.50 \\
6 & 2.23 \\
8 & 2.18 \\
$\vdots$ & $\vdots$ \\
\hline
\end{tabular}

Table 2: Relation between the degree of $V_{i, k}(x), k=1,2$, and the value of $C$ for which robust stability can be proven. Recall that the system is stable for $p>2.165$.

Using this approach, higher degree Lyapunov functions can be constructed, thus reducing the conservatism of searching for only quadratic candidates. In the same way parametric uncertainty can be incorporated in the search. Several examples have been provided to illustrate the benefits of the new approach.

\section{References}

[1] S. Boyd, L. El Ghaoui, E. Feron, and V. Balakrishnan. Linear Matrix Inequalities in System and Control Theory. SIAM, 1994.

[2] M. S. Branicky. Multiple Lyapunov functions and other analysis tools for switched and hybrid systems. IEEE Trans. Automat. Control, 43(4):475-482, 1998.
B. Lennartson. Perspectives and results on the stability and stabilizability of hybrid systems. Proceedings of the IEEE, 88(7):1069-1082, 2000.

[4] A. Hassibi and S. P. Boyd. Quadratic stabilization and control of piecewise-linear systems. In Proceedings of $A C C$, pages 3659-64, 1998.

[5] M. Johansson and A. Rantzer. Computation of piecewise quadratic Lyapunov functions for hybrid systems. IEEE Trans. Automat. Control, 43(4):555-559, 1998.

[6] A. Papachristodoulou and S. Prajna. On the construction of Lyapunov functions using the sum of squares decomposition. In Proceedings of IEEE CDC, pages 3482-3487, 2002.

[7] P. A. Parrilo. Structured Semidefinite Programs and Semialgebraic Geometry Methods in Robustness and Optimization. $\mathrm{PhD}$ thesis, California Institute of Technology, Pasadena, CA, 2000.

[8] S. Pettersson and B. Lennartson. Stability and robustness of hybrid systems. In Proceedings of IEEE CDC, pages 1202-1207, 1996.

[9] S. Prajna, A. Papachristodoulou, and P. A. Parrilo. SOSTOOLS: Sum of squares optimization toolbox for MATLAB. Available from http://www.cds.caltech.edu/sostools and http: //www aut.ee.ethz.ch/ parrilo/sostools, 2002.

[3] R. A. DeCarlo, M. S. Branicky, S. Pettersson, and 